\title{
Splicing factor derived circular RNA circUHRF1 accelerates oral squamous cell carcinoma tumorigenesis via feedback loop
}

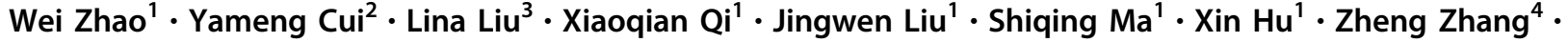 \\ Yue Wang ${ }^{1} \cdot$ Hongfa $\mathrm{Li}^{1} \cdot$ Zuomin Wang $^{5} \cdot$ Zihao Liu $^{1} \cdot$ Jie $\mathrm{Wu}^{1}$
}

Received: 28 May 2019 / Revised: 16 August 2019 / Accepted: 6 September 2019 / Published online: 30 September 2019

(C) The Author(s) 2019

\begin{abstract}
Emerging evidences have suggested the vital roles of circular RNA (circRNA) in the human cancers. However, the underlying biological functions and biogenesis of circRNA in the oral squamous cell carcinoma (OSCC) is still ambiguous. Here, we investigate the oncogenic roles and biogenesis of the novel identified circRNA, circUHRF1 (hsa_circ_0002185), in the OSCC tumorigenesis. Results showed that circUHRF1 was markedly upregulated in the OSCC cells and tissue, besides, the overexpression was closely correlated with the poor prognosis of OSCC patients. Functionally, circUHRF1 promoted the proliferation, migration, invasion, and epithelial mesenchymal transformation (EMT) in vitro and the tumor growth in vivo. Mechanically, circUHRF1 acted as the sponge of miR-526b-5p, thereby positively regulating c-Myc. Transcription factor c-Myc could accelerate the transcription of TGF- $\beta 1$ and ESRP1. Moreover, splicing factor ESRP1 promoted the circularization and biogenesis of circUHRF1 by targeting the flanking introns, forming the circUHRF1/miR-526b-5p/c-Myc/ TGF- $\beta 1 /$ ESRP1 feedback loop. In conclusion, our research identified the oncogenic roles of circUHRF1 in the OSCC tumorigenesis and EMT via circUHRF1/miR-526b-5p/c-Myc/TGF- $\beta 1 / E S R P 1$ feedback loop, shedding light on the pathogenic mechanism of circUHRF1 for OSCC and providing the potential therapeutic target.
\end{abstract}

\section{Introduction}

Oral squamous cell carcinoma (OSCC) acts as one of the most aggressive head and neck cancers, being characterized by the high rates of recurrence and metastasis [1]. The long-

Wei Zhao, Yameng Cui, Lina Liu and Xiaoqian Qi are both first authors

Edited by D. Aberdam

Supplementary information The online version of this article (https:// doi.org/10.1038/s41418-019-0423-5) contains supplementary material, which is available to authorized users.

Zuomin Wang

wzuomin@sina.cn

Zihao Liu

liuzihao@tmu.edu.cn

$\triangle$ Jie Wu

wujiedoctor@tmu.edu.cn

1 The School and Hospital of Stomatology, Tianjin Medical University, 300070 Tianjin, PR China term prognosis and overall 5-year survival rate of OSCC patients is poor due to the distant metastasis and lymphatic metastasis [2]. Although the therapeutic methods have been improved in decades, especially surgical method and chemoradiotherapy, the therapeutic effect is still dissatisfied. Therefore, it is urgently essential to develop new treatments specifically targeting the true pathogenesis.

Circular RNAs (circRNAs) are emerging subgroup of endogenous noncoding RNAs, being characterized by the tissue specificity and structure stability [3, 4]. Compared with the traditional linear RNA, circRNA is generated by the backsplicing to be a covalently closed loop without a $5^{\prime}$-cap or a $3^{\prime}$-poly(A) tail [4-7]. In spite of being neglected for decades,

2 Department of Integrative Oncology, Tianjin Medical University Cancer Institute and Hospital, Tianjin, PR China

3 Department of Prosthodontics, Tianjin Stomatological Hospital, Hospital of Stomatology, NanKai University, 300041 Tianjin, PR China

4 Department of Periodontics, Tianjin Stomatological Hospital, Hospital of Stomatology, NanKai University, 300041 Tianjin, PR China

5 Beijing Chaoyang Hospital, Capital Medical University, Department of Stomatology, 100022 Beijing, PR China 
circRNAs dramatically catch researcher's attention due to their potential important roles in the regulation of pathophysiological process at the transcription or posttranscription. Several circRNAs have been reported to regulate the OSCC tumorigenesis. For example, circRNA_100290 is upregulated in OSCC tissue, sponging miR-29b family to upexpress CDK6 to promote the OSCC progression [8].

The major mechanism for the downstream of circRNA is the competing endogenous RNA [9]. CircRNAs could absorb their targeting miRNAs and indirectly regulate the downstream functional proteins [10]. However, the upstream biogenesis for the circRNA is acquaint scarcely [11]. RNA binding proteins (RBPs) have been validated to interact with RNAs to regulate the gene expression, as well as the circRNA [12]. Alternative splicing factors are group of RBPs in the cells [13]. It has been identified that splicing factor could bind with the flanking intron of exon to accelerate the back-splicing of circRNAs. For example, alternative splicing factor Quaking (QKI) promotes the circRNA biogenesis during epithelial to mesenchymal transition. The QKI motif addition could induce the circRNA formation rather than linearly spliced transcript [14]. Alternative splicing factor could participate in the biogenesis of circRNA via activating the back-splicing, providing as novel insight for the upstream of circRNA.

Epithelial splicing regulatory protein 1 (ESRP1), also named as RNA-binding motif protein 35A (RBM35A), is a critical elements in the human cancer epithelial mesenchymal transformation (EMT) [15-18]. It has been identified that ESRP1 regulate the alternative splicing events associated with epithelial phenotypes in OSCC [19]. ESRP1 and TGF- $\beta 1$ are both vital regulators in the human cancer EMT [20-22]. Furthermore, ESRP1 is validated to be one of EMT-associated splicing factor in tumor metastasis, as well as QKI [14].

Here, we discovered a novel identified circRNA, circUHRF1 (hsa_circ_0002185), in the OSCC cells. circUHRF1 is generated from the 12, 13 exon of UHRF1 gene with genomic location chr19:4950622-4951008. In our cellular functional investigation, we found that circUHRF1 could promote the tumorous malignant behavior of OSCC cells and facilitates the EMT. In this work, we further investigate the oncogenic of circUHRF1 and its deepgoing mechanism in OSCC tumorigenesis. Moreover, we explore the potential RBPs targeting circUHRF1 to illustrate its biogenesis.

\section{Results}

\section{circUHRF1 is highly expressed in the OSCC tissue and cells}

In the preliminary experimental screening, we detected hundreds of potential circRNAs from circBase database (http://www.circbase.org) using RT-PCR (Additional file 4,
Fig. S1). Finally, we found that circUHRF1 (hsa_circ_0002185) was significantly up-regulated in the OSCC cells (Fig. 1a). However, its potential functions were unknown. circUHRF1 was derived from the UHRF1 gene (chr19:4950622-4951008) and generated by its 12, 13 exon via back-splicing (Fig. 1b). The sequence of 12, 13 exon of UHRF1 gene was presented (Additional file 5, Fig. S2). Online CircInteractome tools (https://circinteractome.nia. nih.gov/) showed the conjunction sites of circUHRF1, and the binding sites of 12, 13 exon (Additional file 6, Fig. S3). Sanger sequencing indicated that the 12,13 exon of UHRF1 gene was back-spliced to form the closed loop construction (Fig. 1c). Divergent primers and convergent primers for circUHRF1 and linear transcript were designed. The cDNA and genomic DNA (derived from SCC25 cells) was amplified and analyzed using agarose gel electrophoresis (Fig. 1d, Additional file 7, Fig. S4). To identify the subcellular distribution of circUHRF1 in OSCC cells, RNAFluorescence in situ hybridization (RNA-FISH) was performed using SCC25 and CAL27 cells, indicating that circUHRF1 was mainly located in the cytoplasm (Fig. 1e). After the SCC25 cells were treated with the transcription inhibitor Actinomycin D, the RNA was extracted and analyzed by RT-PCR. Data showed that the transcript half-life of circUHRF1 is significantly longer than the linear transcript, indicating the stability of circUHRF1 (Fig. 1f). RNase $\mathrm{R}$ was administrated to the extracted RNA, illustrating that the circular form (circUHRF1) was able to be resistant to the RNase R, however the linear form (UHRF1 mRNA) was significantly decayed (Fig. 1g). The enrichment of circUHRF1 in the enrolled individuals showed that circUHRF1 was upregulated compared with the normal controls (Fig. 1h). The clinicopathologic data for the OSCC patients was presented (Table 1). The survival rate of OSCC patients was analyzed and indicated that the patients with high level of circUHRF1 had lower survival rate as comparing to these lower ones (Fig. 1i). Overall, in this section, we make the identification for circUHRF1 and illustrated that circUHRF1 is highly expressed in the OSCC tissue and cells.

\section{circUHRF1 modulates the proliferation, migration, invasion, and EMT of OSCC cells in vitro}

In order to identify the potential oncogenic or antioncogenic roles of circUHRF1, functional experiments were performed in vitro using the OSCC cells. OSCC cells (SCC25, CAL27) cells were transfected with the stable silencing shRNA before the assays. Besides, the linear UHRF1 mRNA levels were not regulated by the circUHRF1 shRNA transfection (Fig. 2a). For the proliferation ability, colony formation assay and EdU assay were performed, indicating that knockdown of circUHRF1 inhibited the colony formation ability and DNA 

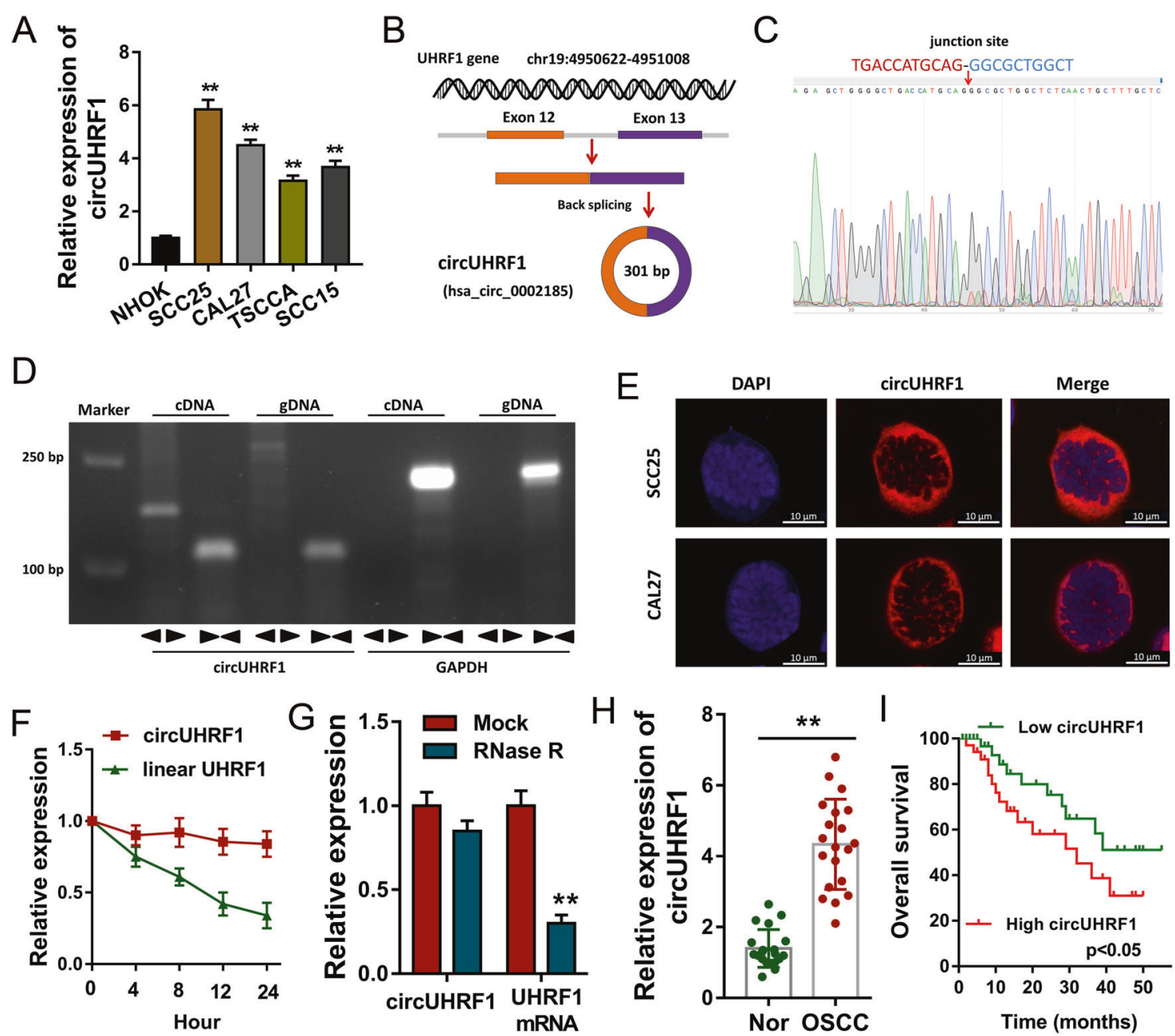

Fig. 1 circUHRF1 is highly expressed in the OSCC tissue and cells. a RT-PCR showed the significant overregulation of circUHRF1 in the OSCC cells. b Schematic diagram presented the formation of circUHRF1 from the 12, 13 exon of UHRF1 gene. c Sanger sequencing identified the existence of circUHRF1. The red arrow indicates the conjunction sites of 12, 13 exon of UHRF1 gene. d The agarose gel electrophoresis was performed using the divergent primers and convergent primers to verify the existence of circUHRF1 using SCC25 cells. e The subcellular distribution of circUHRF1 was identified using RNA-

synthesis (Fig. 2b, Fig. 2c). Wound healing assay illustrated that the knockdown of circUHRF1 repressed the cells migrative ability (Fig. 2d). Transwell assay, including migration and invasion, indicated that the invasive ability and migrative ability was reduced when the circUHRF1 was silenced (Fig. 2e, f). The protein markers of EMT were detected. Western blot analysis showed that the knockdown of circUHRF1 repressed the expression of mesenchymal markers (N-cadherin, Vimentin), while enhanced the epithelial marker (E-cadherin) (Fig. 2g). Moreover, we constructed the overexpression of circUHRF1 (gain-of-functional experiments) in the SCC25 and CAL27 cells, showing that circUHRF1 overexpression promoted the proliferation, migration, invasion, and EMT of OSCC cells (Additional file 8: Fig. S5). In conclusion, the data evidences that knockdown

FISH using SCC25 and CAL27 cells. The red (Cy3-labeled probe) indicated the circUHRF1. DAPI indicated the nucleus. $f$ RT-PCR showed the transcript half-life of circUHRF1 in SCC25 cells treated with transcription inhibitor Actinomycin D. g RNase R was administrated to the extracted RNA to measure the relative expression of circular form (circUHRF1) and the linear form (UHRF1 mRNA). h RT-PCR showed the enrichment of circUHRF1 in the enrolled OSSCC individuals and normal controls. i The survival rate of OSCC patients who with high level of circUHRF1 and lower level. **p-value $<0.01$

of circUHRF1 represses the proliferation, migration, invasion, and EMT of OSCC cells in vitro.

\section{circUHRF1 acts as the sponge of miR-526b-5p in OSCC cells}

Existing theories suggest that these circRNA derived from exons might tend to distribute in the cytoplasm, acting as the miRNA sponge to absorb its target miRNAs [3, 23]. Online bioinformatics tools CircInteractome (https:// circinteractome.nia.nih.gov/) indicated that there are several potential miRNAs with targeting sites for circUHRF1 (Additional file 9: Table S4), meanwhile, miR-526b-5p might act as the target of circUHRF1 (Fig. 3a). For the luciferase reporter assay, the wild-type and mutant 
Table 1 Correlation within circUHRF1 expression and the clinicopathological characteristic of OSCC patients

\begin{tabular}{|c|c|c|c|c|}
\hline \multirow[t]{2}{*}{ Characteristics } & & \multicolumn{2}{|c|}{ circUHRF1 expression } & \multirow[t]{2}{*}{$p$-value } \\
\hline & & Low $=10$ & $\operatorname{High}=10$ & \\
\hline \multicolumn{5}{|l|}{ Gender } \\
\hline Male & 12 & 6 & 6 & 0.587 \\
\hline Female & 8 & 4 & 4 & \\
\hline \multicolumn{5}{|l|}{ Age } \\
\hline$<60$ years & 11 & 5 & 6 & 0.845 \\
\hline$\geq 60$ years & 9 & 5 & 4 & \\
\hline \multicolumn{5}{|l|}{ TNM stage } \\
\hline I-II & 7 & 4 & 3 & $0.008 *$ \\
\hline III-IV & 13 & 6 & 7 & \\
\hline \multicolumn{5}{|l|}{ Tumor size } \\
\hline$<3 \mathrm{~cm}$ & 8 & 5 & 3 & 0.162 \\
\hline$\geq 3 \mathrm{~cm}$ & 12 & 5 & 7 & \\
\hline \multicolumn{5}{|c|}{ Histological differentiation } \\
\hline Well/moderate & 12 & 7 & 5 & 0.286 \\
\hline Poor & 8 & 3 & 5 & \\
\hline
\end{tabular}

${ }^{*} P<0.05$ represents statistical differences

sequences were constructed corresponding with the miR526b-5p (Fig. 3b). The cotransfection of miR-526b-5p and circUHRF1 wild type could decrease the luciferase activity, indicating the binding within miR-526b-5p and circUHRF1 (Fig. 3c). RNA pull-down assay showed that miR-526b-5p was pulled down by the biotinylated-circUHRF1 probe in SCC25 cells (Fig. 3d). The transfection of circUHRF1 shRNA could increase the miR-526b-5p expression level (Fig. 3e). In the OSCC (SCC25, CAL27) cells, RT-PCR illustrated that miR-526b-5p was reduced compared with the control cells (Fig. 3f). To identify the subcellular distribution of circUHRF1 and miR-526b-5p in OSCC cells, RNA-FISH was performed and indicated that circUHRF1 and miR-526b-5p were both located in the cytoplasm (Fig. 3g). In the OSCC individuals, circUHRF1 was negatively correlated with the miR-526b-5p (Fig. 3h). Overall, this finding suggests that circUHRF1 acts as the sponge of miR-526b-5p in OSCC cells.

\section{c-Myc functions as the target of circUHRF1/miR- 526b-5p}

In the further exploration of the downstream target of circUHRF1 and miR-526b-5p, we found that c-Myc might functions as the target. The c-Myc wild type and mutant sequence at $3^{\prime}$-UTR and miR-526b-5p were constructed (Fig. 4a). Luciferase reporter assay indicated the molecular binding for miR-526b-5p with c-Myc (Fig. 4b). To identify the interaction within miR-526b-5p and c-Myc, we transfected the miR-526b-5p mimics and inhibitor into the SCC25 cells. Results by RT-PCR indicated that miR-526b-5p mimics transfection decreased the c-Myc mRNA, while the miR526b-5p inhibitor transfection markedly upregulated the cMyc mRNA (Fig. 4c). Western blot analysis illustrated that miR-526b-5p mimics transfection decreased the c-Myc protein, and the miR-526b-5p inhibitor transfection upregulated the c-Myc protein (Fig. 4d, e). Thus, the data from the RTPCR and western blot draw the same conclusion that miR526b-5p was negatively regulated the c-Myc protein and mRNA in OSCC cells (SCC25, CAL27). In SCC25 cells, the circUHRF1 shRNA transfection repressed the c-Myc protein, while the cotransfection of miR-526b-5p inhibitor recovered it (Fig. 4f, g). Therefore, these finding indicated that c-Myc functions as the target of circUHRF1/miR-526b-5p.

\section{C-Myc promotes the transcription of TGF- $\beta 1$ and ESRP1}

c-Myc is a vital oncogene in the human cancers, besides, it is also a transcription factor participating series of tumorigenesis. Researches and literature have indicated the critical tumorigenesis promoting of c-Myc in the OSCC [24, 25]. In our research, we found that c-Myc not only acted as the target of circUHRF1/miR-526b-5p, but also shared with the binding sites with the promoter region of TGF- $\beta 1$ (Fig. 5a). Two potential binding sites were predicted using the JASPAR (http://jaspar.genereg.net/) and RegRNA (http://regrna 2.mbc.nctu.edu.tw/detection.html). Targeting the two sites, the ChIP-PCR was performed and indicated that the first site $(-129$ to -123$)$ might effectively combine with the c-Myc antibody (Fig. 5b). RT-PCR showed that the c-Myc overexpressed plasmid might enhance the TGF- $\beta 1 \mathrm{mRNA}$ level, while the c-Myc silencing siRNA could reduce it (Fig. 5c). The wild type and mutant sequences of TGF- $\beta 1$ promoter region $(-129$ to -123$)$ were constructed, and luciferase reporter assay indicated that the wild type of this region could combine with the c-Myc, suggesting the c-Mycmediated activation for TGF- $\beta 1$ transcription (Fig. 5d). Moreover, the bioinformatics tools validated that c-Myc also shared with the binding sites with the promoter region of ESRP1 (Fig. 5e). Then, ChIP-PCR indicated that the c-Myc antibody could effectively enrich the first site $(-193$ to -187) of ESRP1 promoter region (Fig. 5f). Similarly, the c-Myc overexpressed plasmid might enhance the ESRP1 mRNA level, while the c-Myc silencing siRNA could reduce it (Fig. 5g). Luciferase reporter assay indicated that c-Myc could bind with the ESRP1 promoter region, suggesting the c-Myc-mediated activation for ESRP1 transcription (Fig. 5h). Western blotting analysis illustrated that c-Myc promoted the expression of TGF- $\beta 1$ and ESRP1 (Fig. 5i). Moreover, western blotting and ELISA showed that circUHRF1 overexpression could upregulate the ESRP1 and TGF- $\beta 1$ levels, while the circUHRF1 silencing reduced their 

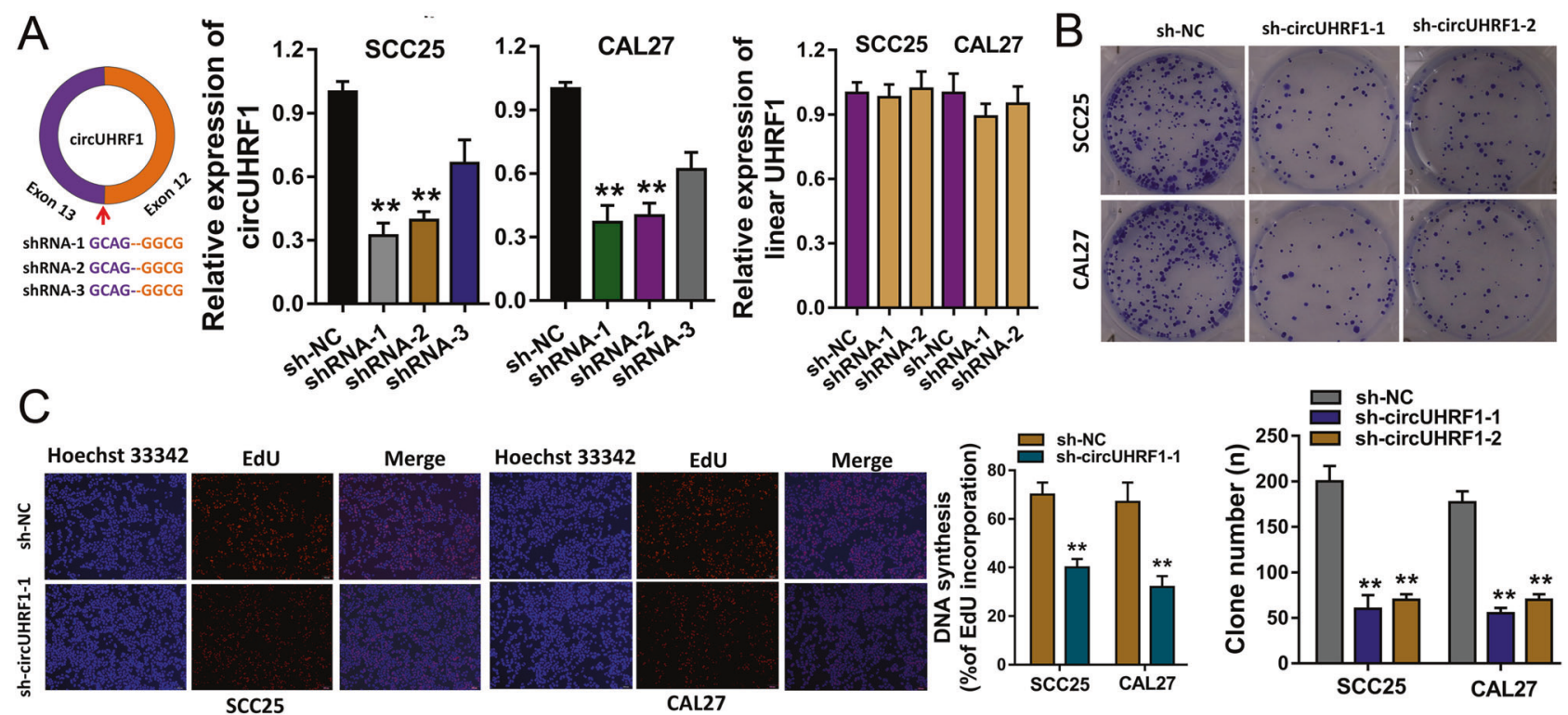

D
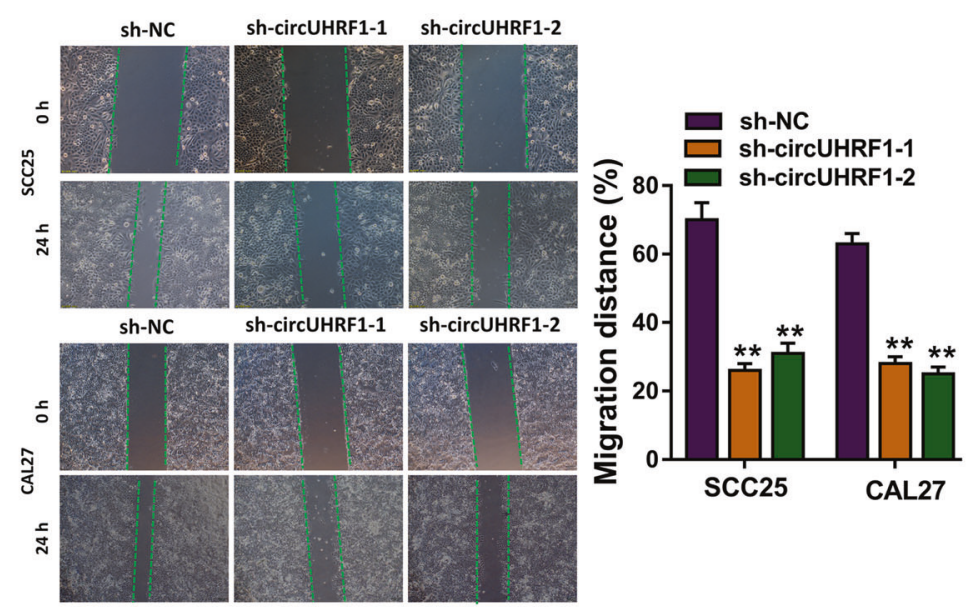

E

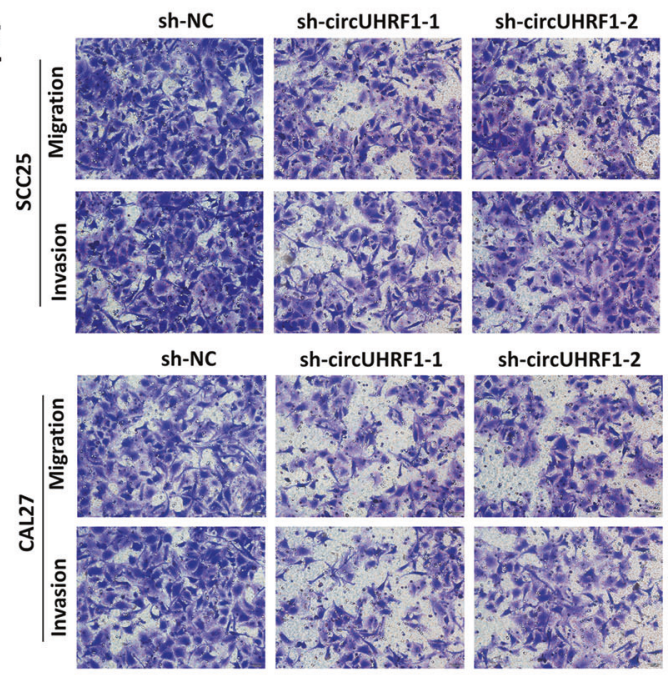

G
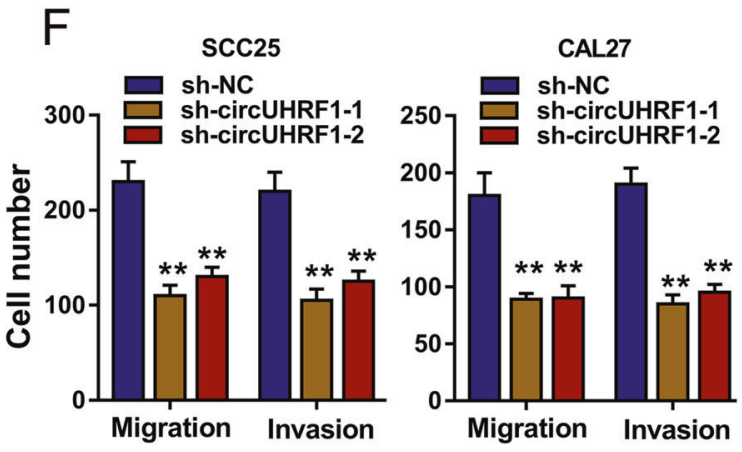

Fig. 2 Knockdown of circUHRF1 represses the proliferation, migration, invasion, and EMT of OSCC cells in vitro. a shRNA targeting circUHRF1 junction sites (Exon 13-Exon 12) were constructed (left). Stable silencing shRNA were transfected into OSCC cells (SCC25, CAL27) to silence circUHRF1 expression (middle). The linear UHRF1 mRNA levels were detected using RT-qPCR (right). b Colony formation assay was performed in the SCC25 and CAL27 cells after stable transfection. $\mathbf{c}$ EdU assay was performed for the DNA synthesis

in the SCC25 and CAL27 cells after stable transfection. d Wound healing assay illustrated migrative ability after the knockdown of circUHRF1 or negative control. e, $\mathbf{f}$ Transwell assay for migration and invasion was carried out to detect the invasive ability and migrative ability of OSCC cells (SCC25, CAL27). $\mathbf{g}$ The protein markers of EMT were detected using western blot analysis, including mesenchymal markers ( $\mathrm{N}$-cadherin, Vimentin) and the epithelial marker (E-cadherin). $* * p$-value $<0.01$ 

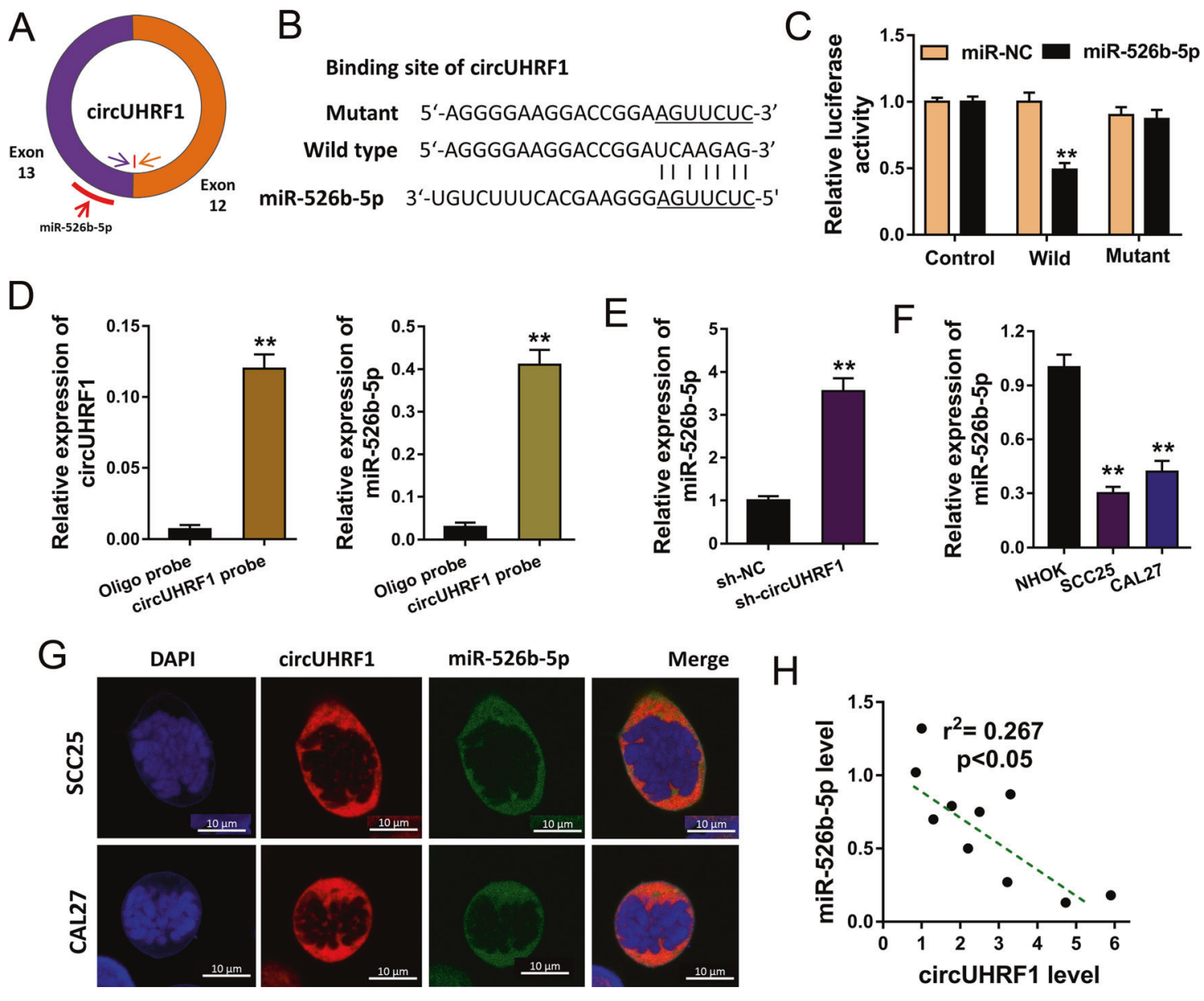

Fig. 3 circUHRF1 acts as the sponge of miR-526b-5p in OSCC cells. a Online bioinformatics tools CircInteractome (https://circinteractome. nia.nih.gov/) indicated the miR-526b-5p target for circUHRF1. b The wild-type and mutant sequences were constructed corresponding with the miR-526b-5p for the luciferase reporter assay. c The luciferase activity in the cotransfection of miR-526b-5p and circUHRF1 wild type or mutant. d Biotinylated-circUHRF1 probe was performed for RNA pull-down assay. RT-PCR revealed the miR-526b-5p expression

levels (Additional file 10: Fig. S6). According the public GEPIA dataset based on the TCGA (http://gepia.cancer-pku. cn/index.html), the expression of c-Myc was positively correlated with that of TGF- $\beta 1$ and ESRP1 (Fig. 5j). Besides, the c-Myc was correlated with the poor prognosis of head and neck cancer patients, and TGF- $\beta 1$ and ESRP1 were high expressed in patients' cohort (Additional file 11: Fig. S7). In the enrolled OSCC patients, we found that the expression of circUHRF1 was positively correlated with that of TGF- $\beta 1$ and c-Myc (Additional file 12: Fig. S8). As is well-known, TGF- $\beta 1$ acts as the vital element of the TGF$\beta 1 /$ Smad signaling pathway, which facilitates the EMT of tumorigenesis. Therefore, these finding suggests that c-Myc promotes the transcription of TGF- $\beta 1$ and ESRP1 in OSCC cells (Fig. 5k). In conclusion, c-Myc is verified to promote the transcription of TGF- $\beta 1$ and ESRP1.

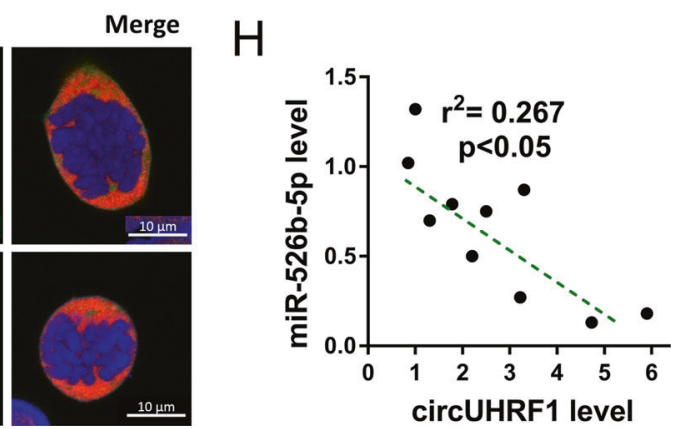

in the pulled down SCC25 cells. e RT-PCR indicated the miR-526b-5p level in the SCC25 cells with transfection of circUHRF1 shRNA. f RT-PCR illustrated the miR-526b-5p level in the OSCC (SCC25, CAL27) cells. g RNA-FISH indicated the circUHRF1 and miR-526b$5 p$ subcellular location. $\mathbf{h}$ The Pearson correlation analysis indicated the negative correlation within miR-526b-5p and circUHRF1. $* * p$-value $<0.01$

\section{Splicing factor ESRP1 accelerates the biogenesis of circUHRF1}

To integrally identify the regulating mechanism of circUHRF1, we next investigated the biogenesis of circUHRF1 in OSCC cells. Splicing factor ESRP1 is a subgroup of RBPs, which had been confirmed to mediate the circularization of circRNAs, such as circANKS1B and circBIRC6 $[26,27]$. We found that there were multiple ESRP1 motifs (GGT-rich) in the flanking of circUHRF1 (Fig. 6a). The minigenes of these motifs were constructed, including wild type and mutant. RNA-binding protein immunoprecipitation (RIP) presented that ESRP1 could bind with the wild type of these sites minigenes, instead of the mutant ones (Fig. 6b). Subsequently, the silenced ESRP1 or controls were transfected with the six mutant motif sites, indicating that ESRP1 knockdown 

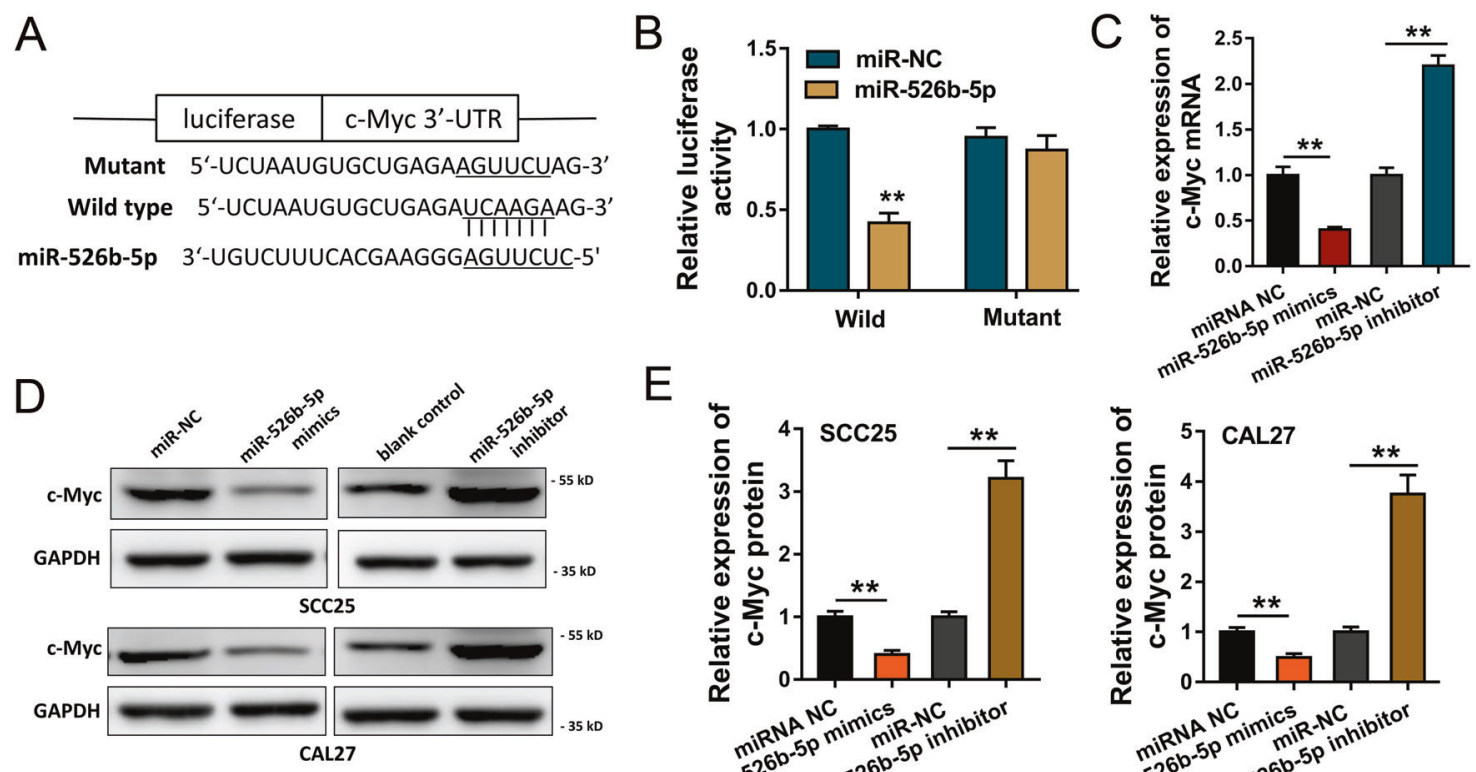

$\mathrm{E}$
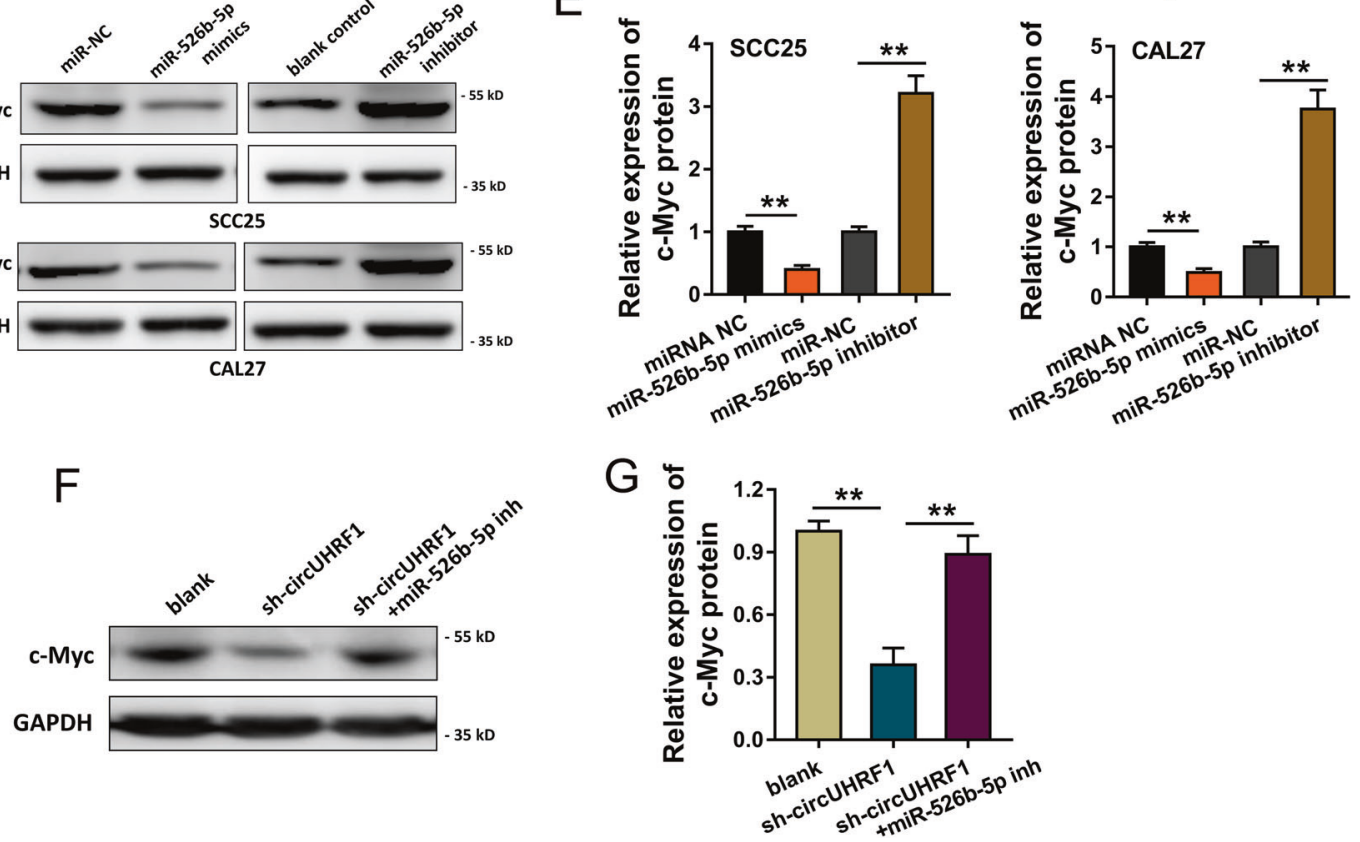

Fig. 4 c-Myc functions as the target of circUHRF1/miR-526b-5p. a The c-Myc wild type and mutant sequence at 3 '-UTR and miR-526b$5 \mathrm{p}$ were constructed. b Luciferase reporter assay indicated the luciferase activity after the cotransfection with c-Myc wild type or mutant and miR-526b-5p mimics or controls. $\mathrm{c}$ RT-PCR indicated the c-Myc mRNA after miR-526b-5p mimics transfection or miR-526b-5p

inhibitor transfection. d, e Western blot analysis illustrated the c-Myc protein in OSCC cells (SCC25, CAL27) with miR-526b-5p mimics or inhibitor transfection. $\mathbf{f}, \mathbf{g}$ Western blot analysis indicated the c-Myc protein in the transfection of circUHRF1 shRNA with miR-526b-5p inhibitor. ${ }^{*} p$-value $<0.01$

could reduce the circUHRF1 expression in these mutant sequences (Fig. 6c, Additional file 13: Fig. S9). Furthermore, when the two motifs $(\mathrm{A} / \mathrm{B}, \mathrm{B} / \mathrm{C}, \mathrm{C} / \mathrm{A}$ in 11 intron, or $\mathrm{D} / \mathrm{E}, \mathrm{E} /$ $\mathrm{F}, \mathrm{F} / \mathrm{D}$ in 13 intron) were mutated at the same time, the results indicated that ESRP1 knockdown could also reduce the circUHRF1 expression (Fig. 6d). However, when three motifs $(\mathrm{A} / \mathrm{B} / \mathrm{C}$ in 11 intron, or $\mathrm{D} / \mathrm{E} / \mathrm{F}$ in 13 intron) were mutated, ESRP1 knockdown could not reduce the circUHRF1 expression. These data indicated that ESRP1 could evenly target these motifs to accelerate the circUHRF1 biogenesis. Moreover, the RT-PCR showed that, after the ESRP1 knockdown, the linear transcript expression levels (exon 12-13, exon 11-12, and exon 13-14) were increased comparing with the ESRP1 control transfection (Fig. 6e). In conclusion, these data support that splicing factor ESRP1 accelerates the biogenesis of circUHRF1 via targeting the flanking intron (Fig. 6f).

\section{circUHRF1/miR-526b-5p/c-Myc/ESRP1 regulates the OSCC tumor behavior}

To identify the role of circUHRF $1 / \mathrm{miR}-526 \mathrm{~b}-5 \mathrm{p} / \mathrm{c}-\mathrm{Myc} /$ ESRP1 axis for the OSCC malignant phenotype, rescue experiments were performed, including transwell assay (Fig. 7a, b), EdU assay (Fig. 7c), and western blot (Fig. 7d). The transfection of miR-526b-5p inhibitor could enhance the invasion, migration, DNA synthesis, and EMT characteristic compared with blank vector. Then, the cotransfection of sh-circUHRF1 or si-c-Myc could recover the phenotype. Moreover, the ESRP1 overexpression could accelerate the invasion, migration, DNA synthesis and EMT characteristic, while the cotransfection of sh-circUHRF1 rescued these characteristic. Thus, these findings identify the role of circUHRF $1 / \mathrm{miR}-526 \mathrm{~b}-5 \mathrm{p} / \mathrm{c}-$ Myc/ESRP1 axis for the OSCC tumor behavior. 
A

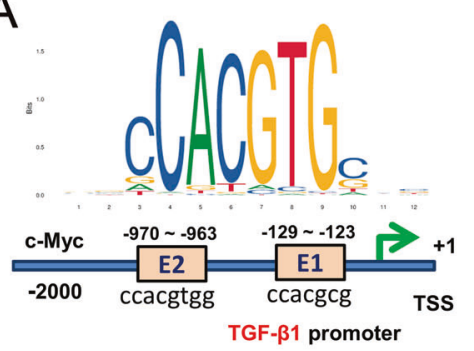

D

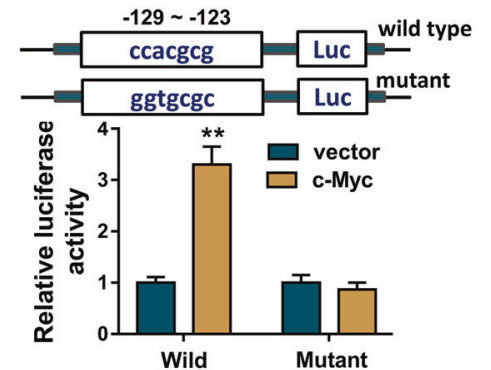

B

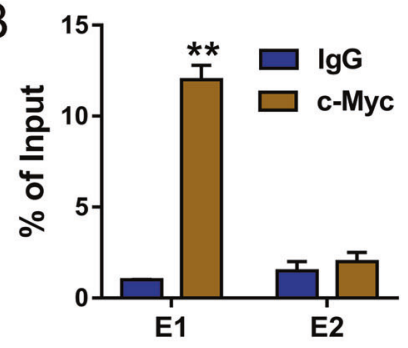

$E$

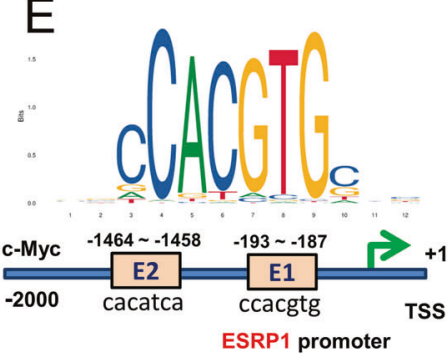

C

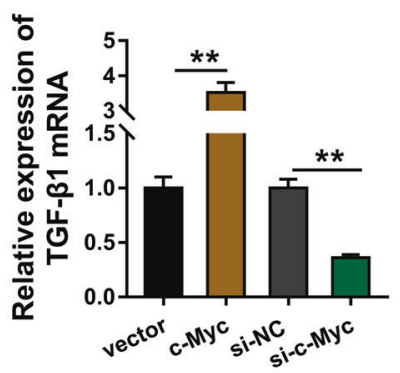

$\mathrm{F}$

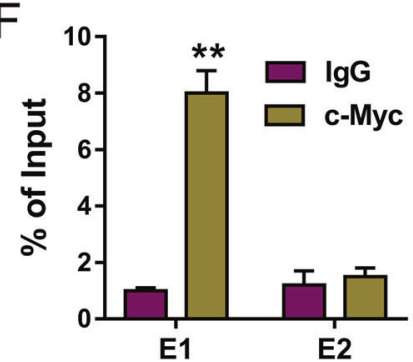

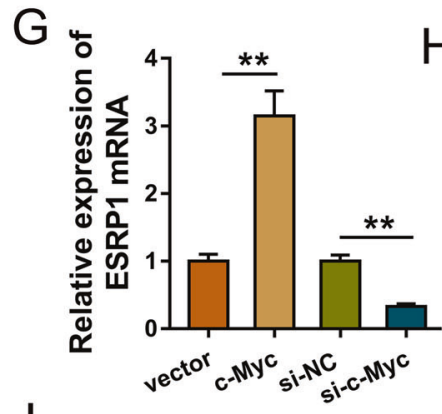

H
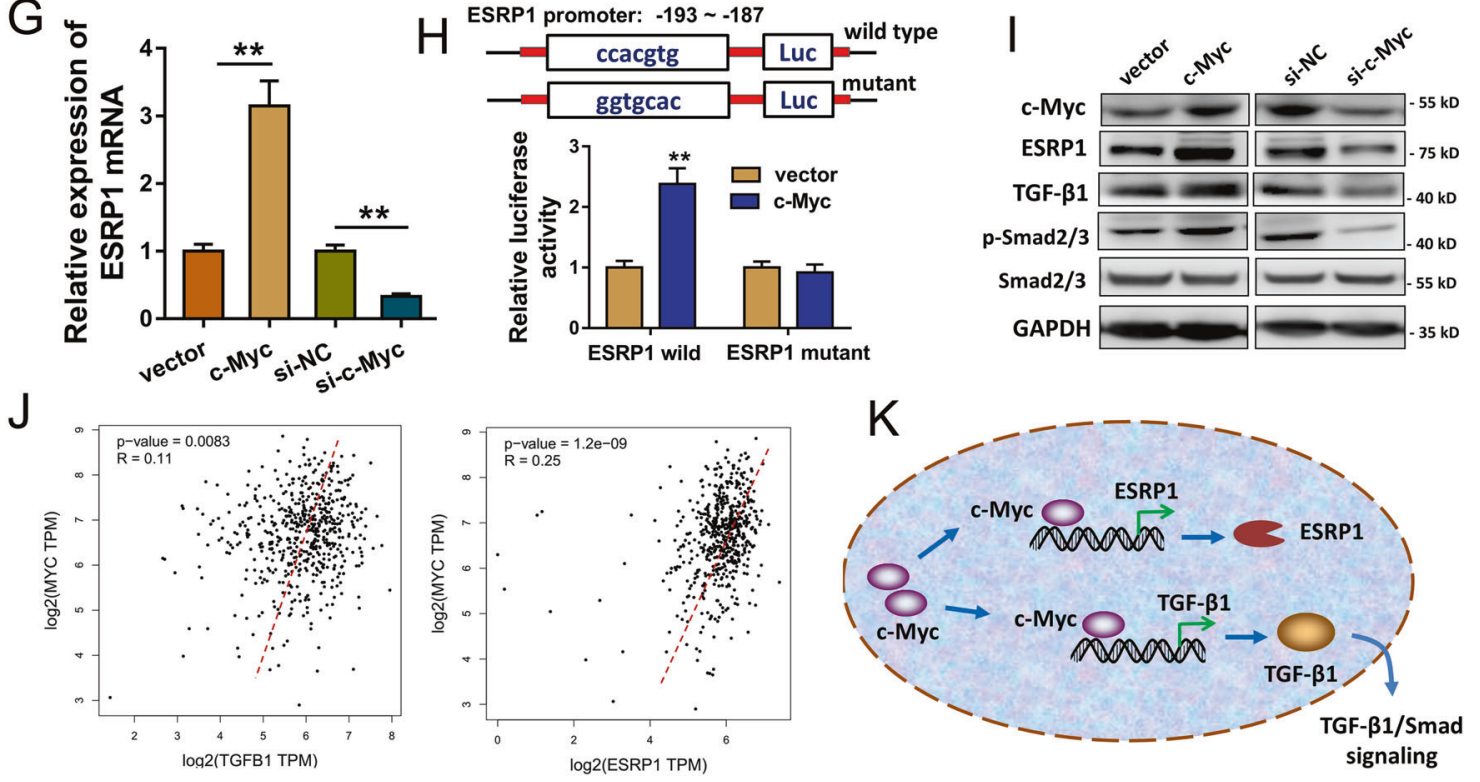

Fig. 5 c-Myc promotes the transcription of TGF- $\beta 1$ and ESRP1. a JASPAR (http://jaspar.genereg.net/) and RegRNA (http://regrna2. mbc.nctu.edu.tw/detection.html) indicated that c-Myc shared with the binding sites with the promoter region of TGF- $\beta 1$. b ChIP-PCR was performed in SCC25 cells to detect the enrichment of potential binding sequences using the c-Myc antibody. IgG acts as the negative control. c RT-PCR showed the TGF- $\beta 1$ mRNA level in SCC 25 cells transfected with c-Myc overexpressed plasmid orc-Myc silencing siRNA. d The luciferase activities were tested in the luciferase reporter assay in SCC25 cells after the vector (wild type and mutant) and c-Myc. e Bioinformatics tools indicated that c-Myc shared with the binding sites with the

promoter region of ESRP1. $\mathbf{f}$ ChIP-PCR showed the binding of c-Myc with the promoter region of ESRP1. g RT-PCR showed the ESRP1 mRNA level with c-Myc overexpressed plasmid orc-Myc silencing siRNA transfection. $\mathbf{h}$ The luciferase reporter assay indicated the binding of the wild type or mutant with c-Myc. i Western blotting analysis for the TGF- $\beta 1$ and ESRP1 protein level after the transfection of c-Myc overexpression plasmid and siRNA. $\mathbf{j}$ The positive correlation within c-Myc and TGF- $\beta 1$ and ESRP1 based on the GEPIA dataset based on the TCGA (http://gepia.cancer-pku.cn/index.html). $\mathbf{k}$ Schematic diagram suggested the activation of c-Myc for the transcription of TGF- $\beta 1$ and ESRP1 in OSCC cells. **p-value $<0.05$

\section{Knockdown of circUHRF1 inhibits the OSCC tumor growth in vivo}

The circUHRF1 stably silencing transfection was conducted for the xenograft in vivo assay. Results indicated that circUHRF1 knockdown significantly inhibited the tumor volume and weight of OSCC cells (Fig. 8a, b). Moreover, the SCC25 cells were transfected with the vectors labeled with firefly luciferase and monitored using a bioluminescence in vivo imaging system. The bioluminescence image showed that circUHRF1 knockdown reduced the tumor metastasis compared with those in the control group 

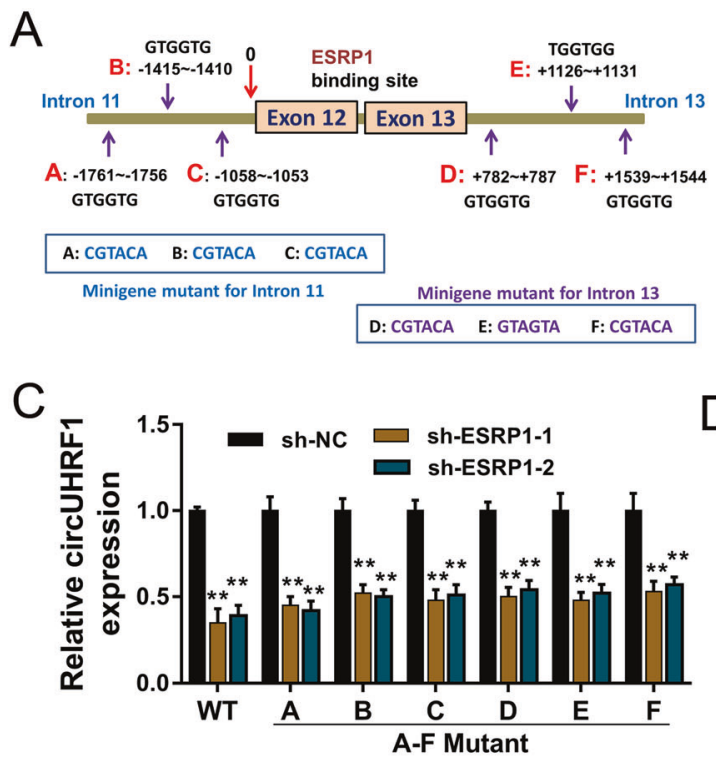

E

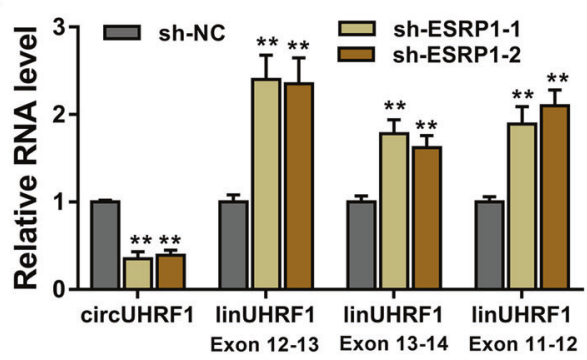

Fig. 6 Splicing factor ESRP1 accelerates the biogenesis of circUHRF1. a Multiple ESRP1 motifs (GGT-rich) were found in the flanking of circUHRF1. The minigenes of these motifs were constructed, including wild type and mutant. b RNA-binding protein immunoprecipitation (RIP) presented the binding of ESRP1 with the motifs minigenes, including wild type and mutant. c RT-PCR showed the circUHRF1 expression in SCC25 cells transfected with

(Fig. 8c). immumohistochemical (IHC) staining and HE staining of the tumor showed that circUHRF1 knockdown reduced the tumor phenotypes (Fig. 8d). As regarding the lung metastasis, no significant lung metastasis was observed within the circUHRF1 knockdown group and control group (Additional file 14: Fig. S10). Taken together, the in vivo assay indicates that knockdown of circUHRF1 inhibits the OSCC tumor growth.

\section{Discussion}

With the rapid development of next-generation sequencing and bioinformatics technology, the critical roles of circRNAs in the human tumorigenesis have been explicated [28-30]. Besides, the biogenesis of circRNAs and its possible mechanisms similarly catch researcher's attention [31]. However, the detailed research
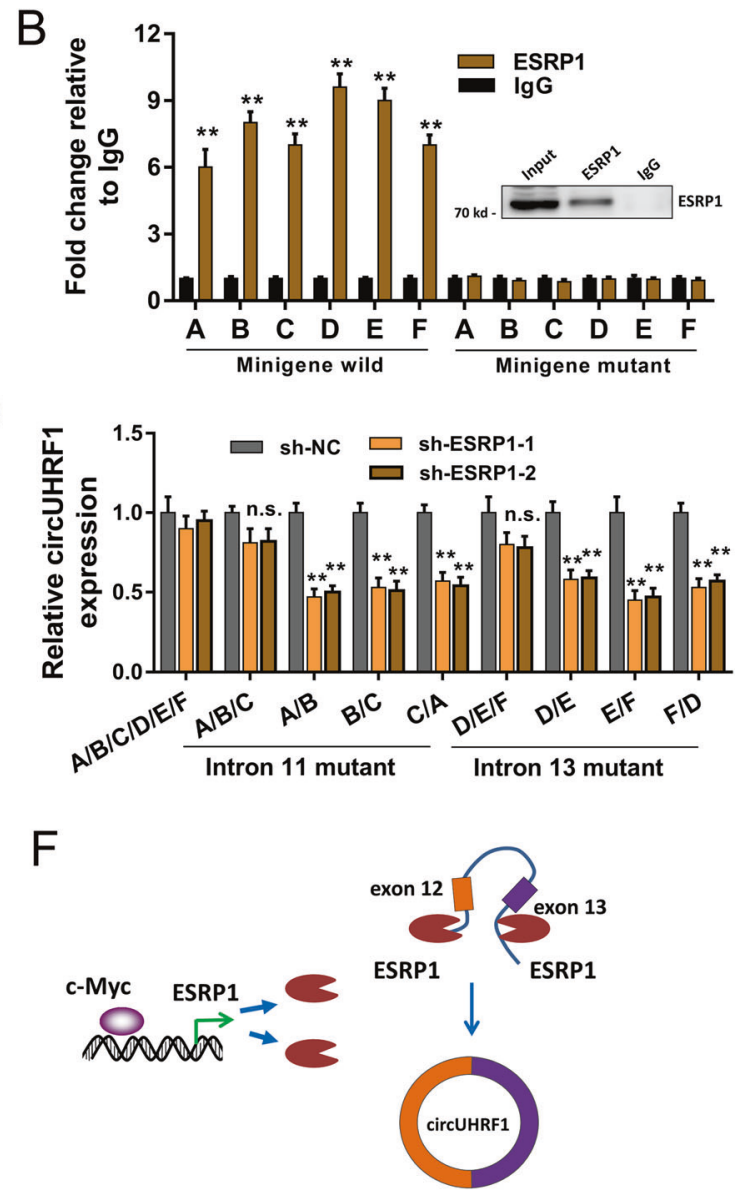

ESRP1 silencing shRNA or controls with these mutant sequences (one motif mutant). d RT-PCR showed the circUHRF1 expression when the two or three motifs were mutated. e RT-PCR showed the linear transcript expression levels (exon 12-13, exon 11-12, and exon 13-14) after the ESRP1 knockdown. f Schematic diagram illustrates that Splicing factor ESRP1 accelerates the biogenesis of circUHRF1 via targeting the flanking intron. ${ }^{* *} p$-value $<0.05$

about circRNAs in the OSCC tumorigenesis is still unclear [32].

The landscape of circRNAs in the human is characterized by tissue specificity and structure stability [33]. The generation of circRNAs is different from that of linear transcript. The transcriptome is spliced into covalent closed loops by the back-splicing. In our initial investigation, we screened the potential circRNAs in the OSCC cells using the largescale RT-PCR screening. Finally, we identified several dysregulated circRNA in the OSCC cells, especially hsa_circ_0002185 (5.85-fold). The novel upregulated circRNA was derived from the exon 12, 13 of UHRF1 gene, thereby being named circUHRF1. The genomic location for circUHRF1 is chr19:4950622-4951008, and the spliced length is $301 \mathrm{bp}$. The validation assay indicated that circUHRF1 had the classical covalent closed loops, which is totally different from the linear RNA. Functional experiments presented that the knockdown of circUHRF1 significantly 
A
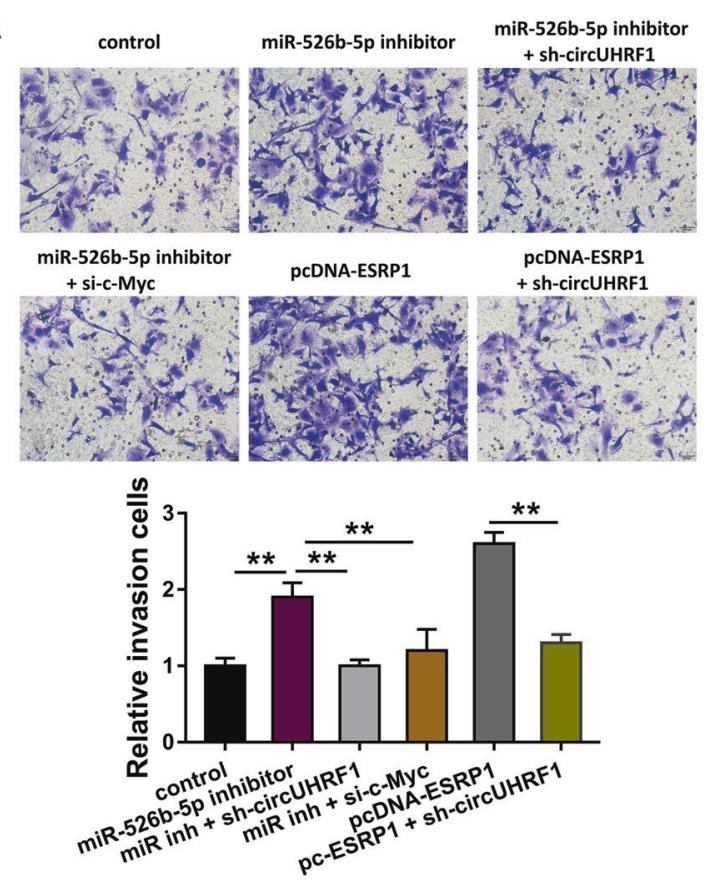

C

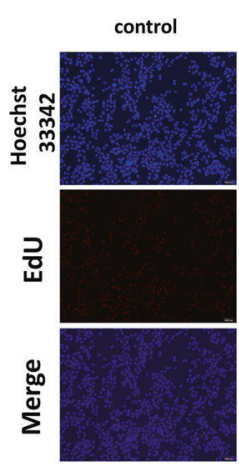

miR-526b-5p inh
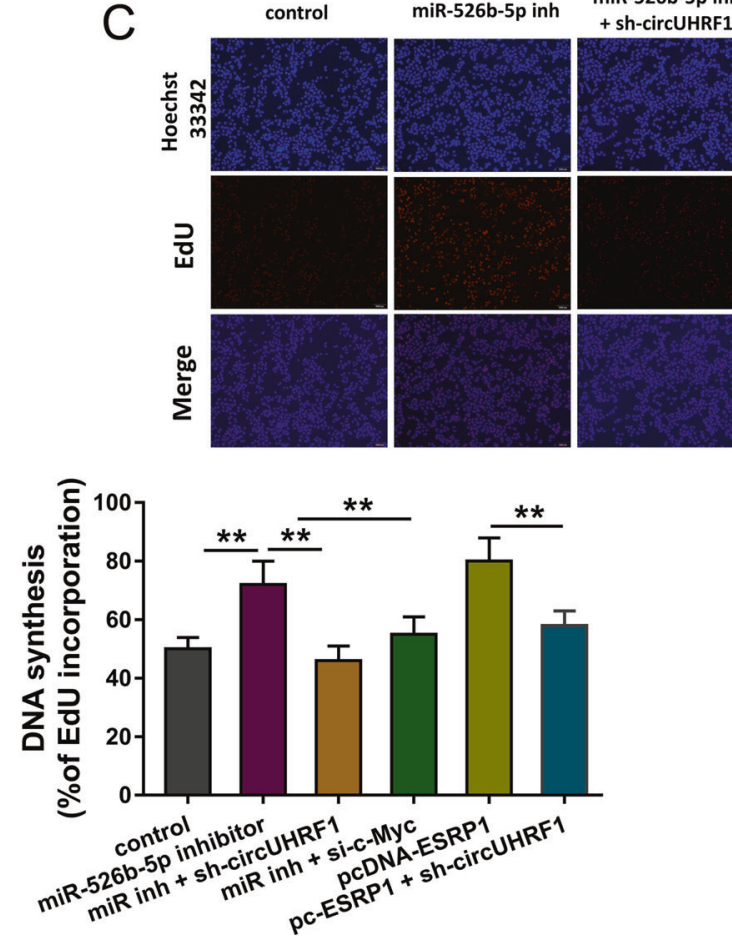

Fig. 7 circUHRF1/miR-526b-5p/c-Myc/TGF- $\beta 1 / E S R P 1$ feedback loop regulates the OSCC metastasis. $\mathbf{a}, \mathbf{b}$ The invasive and migrative ability of SCC25 cells was tested using the transwell assay with the transfection of miR-526b-5p inhibitor, circUHRF1 shRNA (sh-circUHRF1), c-Myc siRNA (si-c-Myc), and ESRP1 pcDNA plasmid (pcDNA-ESRP1).

inhibited the proliferation, migration, invasion, and EMT in vitro, besides repressed the tumor growth in vivo. The data support the oncogenic role of circUHRF1 in the OSCC tumorigenesis.
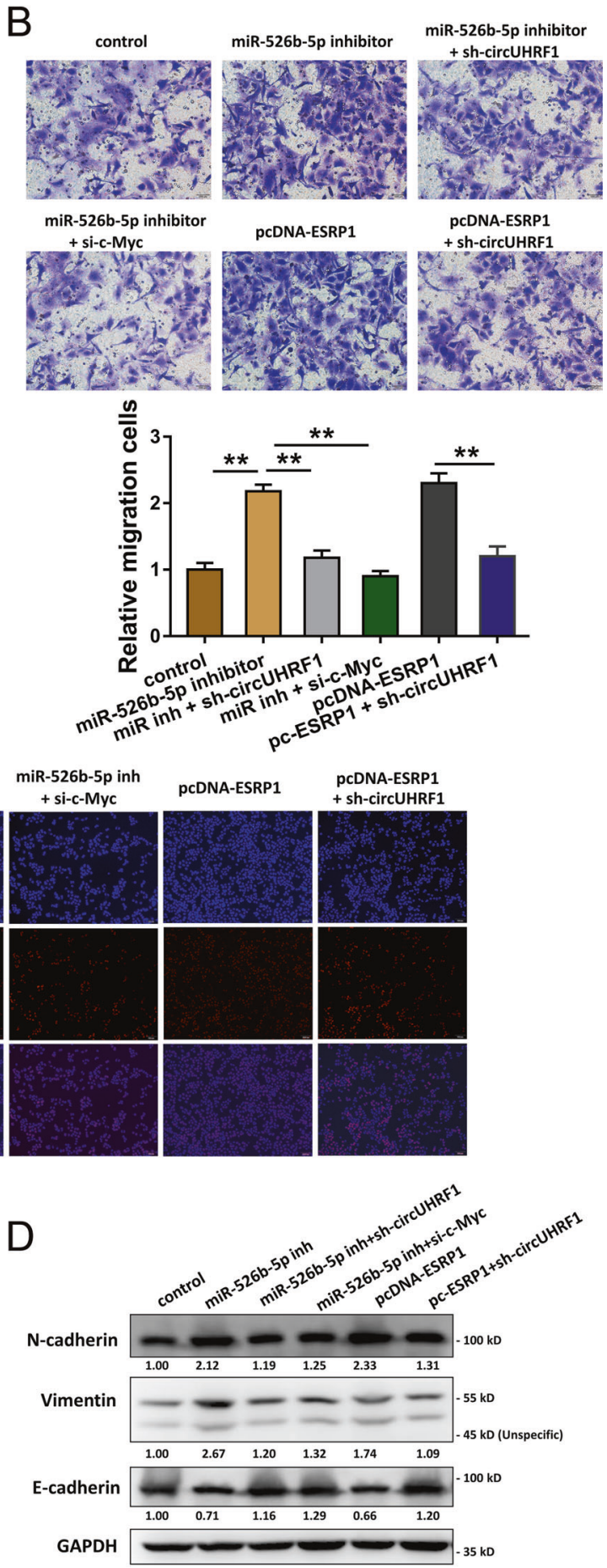

c The DNA synthesis measured by EdU assay. $\mathbf{d}$ The protein markers of EMT were detected using western blot analysis, including mesenchymal markers (N-cadherin, Vimentin) and the epithelial marker (E-cadherin). $* * p$-value $<0.01$

In the mechanical investigation, we found that miR526b-5p acted as the target of circUHRF1, suggesting the miR-526b-5p sponge for circUHRF1. Furthermore, the transcription factor c-Myc was identified to the target of 

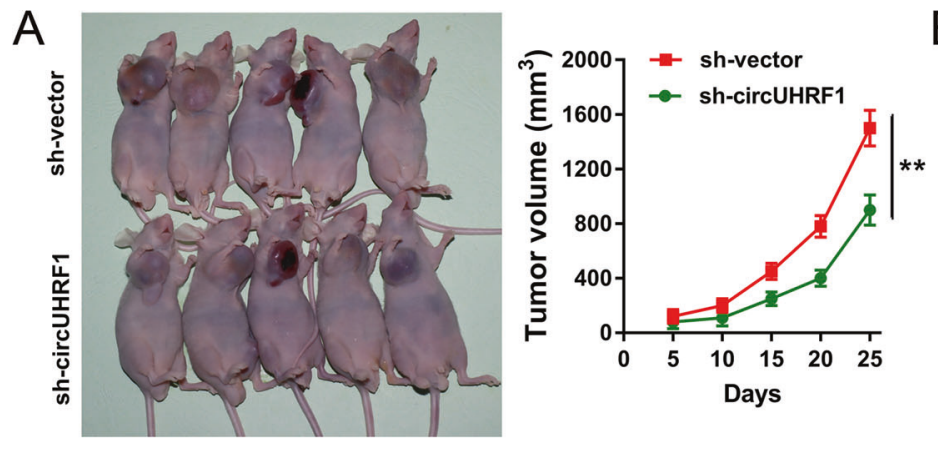

B
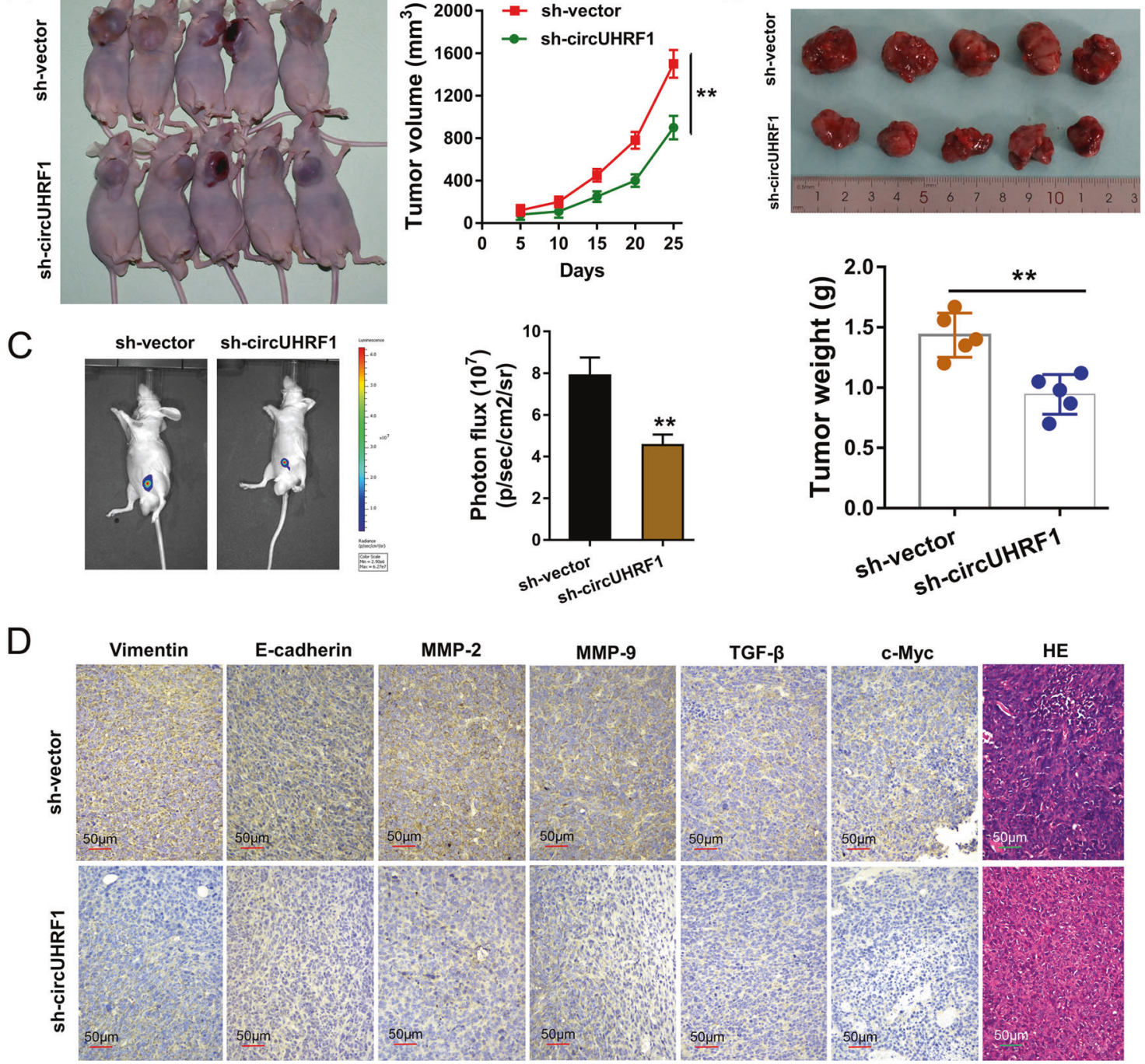

Fig. 8 Knockdown of circUHRF1 inhibits the OSCC tumor growth in vivo. a The tumor volume of mice injected with circUHRF1 knockdown and control group. b The tumor weight in circUHRF1 knockdown and control group. $\mathbf{c}$ The luciferase was monitored using a bioluminescence in vivo imaging system. Bioluminescence image showed the tumor metastasis. d IHC staining and HE staining of the tumor showed that circUHRF1 knockdown reduced the tumor phenotypes. $* * p$-value $<0.01$
miR-526b-5p. Thus, these data supported the circUHRF1/ $\mathrm{miR}-526 \mathrm{~b}-5 \mathrm{p} / \mathrm{c}-\mathrm{Myc}$ axis. It has been confirmed that c-Myc is a critical oncogene and transcription factor in the OSCC [24, 34]. Furthermore, transcription factor c-Myc could promote the transcription level of TGF- $\beta 1$ and ESRP1. Interestingly, the ESRP1, a splicing factor for the EMT progression, was identified to target the flanking introns of circUHRF1 and accelerate its circularization, constructing the circUHRF1/miR-526b-5p/c-Myc/TGF- $\beta 1 / E S R P 1$ feedback loop (Fig. 9) in the EMT progression.

As well known, the dominating circRNAs are generated from the exons of genes which are also transcribed to linear mRNA. The most common circRNAs are formed by $2-3$ exons, meanwhile the internal introns are removed. This raises a question that what type of exons could be efficiently spliced to be circRNA. In the screening, we noticed an interesting tendency that these exons, who are accompanied by extraordinarily long flanking introns, could be cyclized more easily. The reason for this phenomenon is that there are multiple complementary repeating sequences within the flanking intron region, which could promote the circularization of exons by RBPs. The characteristic rules for the circRNA biogenesis inspire us that researchers could explore and forecast the underlying novel circRNA through searching the complementary sequence in the flanking intron region.

The discovery process and biogenesis of circUHRF1 is in conformity to the principles. Primarily, there are extraordinarily long flanking introns (11, 13 introns) for 12, 13 exons (more than $3000 \mathrm{bp}$ ). Besides, there are multiple 


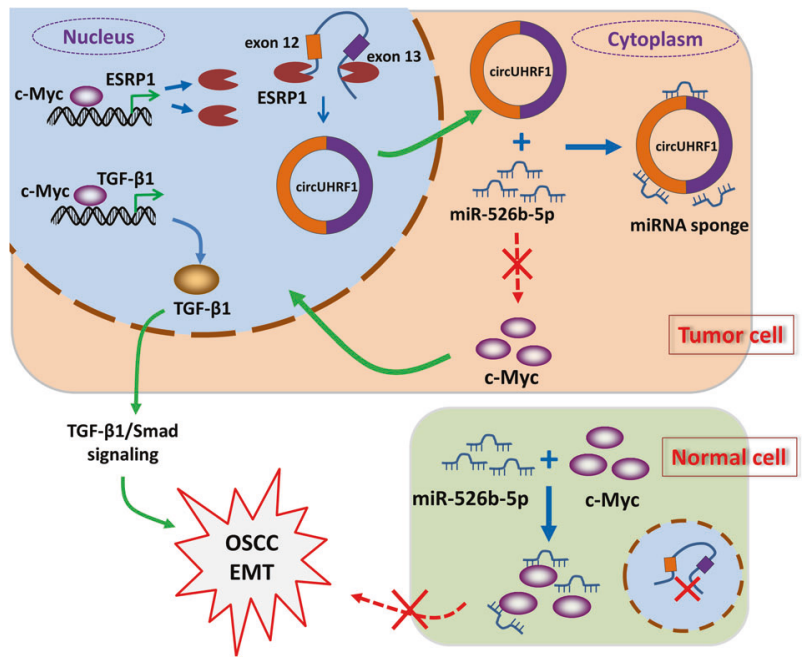

Fig. 9 circUHRF1/miR-526b-5p/c-Myc/TGF- $\beta 1 / E S R P 1$ feedback loop regulates the OSCC cell's EMT

repeating sequences (GGT-rich region, the target of splicing factor ESRP1) in the flanking introns (11, 13 introns). Moreover, the intron (12 intron) within the 12,13 exons is particularly short ( $85 \mathrm{bp}$ ), thereby easily being removed. Therefore, these structural features determine the circularization of circUHRF1. Another interesting phenomenon we noticed was that several reported circRNAs were derived from the E3 Ubiquitination ligase, such as circ-ITCH [35], circRNA CUL2 (circ-10720) [36]. Similarly, circUHRF1 is derived from an E3 Ubiquitination ligase UHRF1 [37]. However, there is still not any powerful evidence to support this point, which needs further validation.

As regarding the biogenesis of circRNAs, existing studies have demonstrated that multiple factors could regulate the biogenesis of circRNA from the precursor-mRNA, such as alternative splicing factors QKI [14], muscleblind [38], eukaryotic translation initiation factor 4A3 [39], transcription factor Twist1 [36], and YY1 super-enhancer [40]. ESRP1 acts as a vital splicing factor participating the biogenesis of circRNAs. For example, splicing factor ESRP1 promotes the circBIRC6 biogenesis in the human embryonic stem cell [27]. In breast cancer, splicing factor ESRP1 promotes the circANKS1B biogenesis [26]. In our research, we discovered that splicing factor ESRP1 could also promote the biogenesis of circUHRF1. We found that ESRP1 shared the GGT-rich region with the flanking intron of 12, 13 exon of UHRF1, thus it could be cyclized at multiple sites. We notice that there are similar binding sites on the ESRP1 promoter and TGF- $\beta 1$ promoter region. The transcription factor c-Myc could both bind with the promoter region of ESRP1 and TGF- $\beta 1$. Interestingly, we found that this functional region on ESRP1 and TGF- $\beta 1$ was also targeted by transcription factor USF1, which had been reported by Zeng K et al. (2018) [26] in breast cancer.
Our findings evidence the regulating function of ESRP1 and TGF- $\beta 1$ in the OSCC EMT tumorigenesis.

Taken together, our research identified the novel circRNA circUHRF1 in the OSCC tumorigenesis and EMT. Besides, the ectopic overexpression of circUHRF1 was closely correlated with the poor prognosis of OSCC patients. Moreover, we validated that the circUHRF1/miR526b-5p/c-Myc/TGF- $\beta 1 /$ ESRP1 feedback loop contributed the OSCC EMT progression, shedding light on the pathogenic mechanism of circRNA for OSCC, and providing the potential therapeutic target.

\section{Materials and methods}

\section{Clinical tissue samples collection and ethic}

This research had been approved by The Hospital of Stomatology, Tianjin Medical University. Each volunteer had minutely acquainted the announcements and signed the informed consent. Twelve tissue specimens and their matched adjacent normal tissue were collected during the surgical excision. Tissue were rapidly frozen at $-80^{\circ} \mathrm{C}$ and also histopathologically confirmed by pathologists. None of these received pharmic or radiative anticancer treatment.

\section{Cell lines}

The OSCC cell lines (SCC25, CAL27, SCC15, and TSCCA) were provided by ATCC and cultured in DMEM medium (Gibco, Carlsbad, CA, USA) supplemented with $10 \%$ fetal bovine serum, $100 \mathrm{U} / \mathrm{mL}$ penicillin, and $100 \mathrm{mg} /$ $\mathrm{mL}$ streptomycin. The normal oral keratinocytes was provided by Institute of Biochemistry and Cell Biology of the Chinese Academy of Sciences (Shanghai, China). The culture were set with humidified air at $37^{\circ} \mathrm{C}$ with $5 \% \mathrm{CO}_{2}$.

\section{Transfection}

The shRNAs targeting circUHRF1 and ESRP1 were subcloned into pLKD-CMV-EGFP vectors, as well the overexpression plasmids for circUHRF1, were synthesized by GeneWiz Biotech (Guangzhou, China). The miR-256b-5p mimic and inhibitor were synthesized by RiboBio Biotech (Guangzhou, China). The transfection was conducted by using Lipofectamine 3000 reagent (Invitrogen, Carlsbad, USA) according to the manufacturer's instruction.

\section{Quantitative real-time PCR (qRT-PCR)}

Total RNA was extracted from OSCC tissues and cells using TRIzol reagent (Invitrogen, CA, USA) based on the manufacturer's protocols. The administration (Actinomycin 
D, RNase R) followed by qRT-PCR for RNA isolation (circular form, linear form) was performed as previously described [41]. The cDNA of circUHRF1 and mRNA were synthesized by using reverse transcription PrimeScript RT Master Mix (Takara, Dalian, China). The cDNA of miR526b-5p was synthesized by using RiboBio reverse transcription kit (Guangzhou, China) following the manufacturer's protocol. Quantification of circUHRF1, miR526b-5p, and mRNA were performed by using a SYBR Green PCR Kit (Takara or RiboBio). The level of circUHRF1 and mRNA were normalized to the control GAPDH. The level of miR-526b-5p was normalized to the control U6. Relative expression was calculated using the $2^{-\triangle \triangle \mathrm{Ct}}$ method. The primer sequences were listed in Additional file 1: Table S1.

\section{RNA-Fluorescence in situ hybridization}

FISH was performed according to the manufacturer's protocol (GenePharma, Shanghai, China). Hybridization of circUHRF1 was administrated by Cy3-labeled probe, and the miR-526b-5p was administrated by FAM-labeled probe. The FISH staining was performed according to the manufacturer's instruction. 4,6-diamidino-2-phenylindole (DAPI) was administrated to stain the cell nucleus. The subcellular distribution of circUHRF1 and miR-526b-5p in OSCC cells was observed with a confocal laser scanning microscope (Olympus FV1000).

\section{Sanger sequencing}

RNA was extracted from OSCC cells and the cDNA was reversely transcribed using the RNA. Sanger sequencing was performed by the Synbio biotech company (Soochow, China).

\section{Edu and colony formation assay}

The proliferation potential of OSCC cells was assessed using EDU and colony formation assay. For the EDU, the assay was conducted using EdU Apollo DNA in vitro kit (RIBOBIO, Guangzhou, China) following the manufacturers' instructions. The results were detected under an immunofluorescence microscope. For the colony formation assay, OSCC cells (SCC25, CAL27) were seeded into the six-well plates at the destiny of one thousand cells per well. After 14 days of culture, cells were fixed with paraformaldehyde and stained by $0.1 \%$ crystal violet.

\section{Western blotting analysis}

Proteins were extracted by RIPA lysis buffer (Thermo Scientific, Rockford, IL, USA) and the concentration was determined using BCA Protein assay kit (Beyotime). Proteins were separated by electrophoresis and then transferred to PVDF membrane for the incubation with primary antibodies, including anti-E-cadherin (ab40772, Abcam), antiN-cadherin (ab18203, Abcam), anti-Vimentin (ab137321, Abcam), anti-c-Myc (ab56, Abcam), anti-TGF- $\beta 1$ (ab92486, Abcam), anti-Smad2/3 (ab202445, Abcam), anti-phosphorylated Smad2/3 (ab63399, Abcam), and antiESRP1 (21045-1-AP, Proteintech). GAPDH (ab8245, Abcam) acted as the control. After incubation overnight, membranes were blocked and administrated with HRPconjugated secondary antibodies at room temperature. Finally, the blots were detected using an enhanced chemiluminescent kit (Thermo Fisher Scientific).

\section{Wound healing and transwell assay}

Monolayer OSCC cells were seeded in six-well plate and then scratched with a sterile $200 \mu$ l pipette tip, and then cultured in serum-free medium.. After 0 and $24 \mathrm{~h}$, the width of wounds was measured, respectively. Transwell assay was performed for the migration and invasion of OSCC cells (SCC25, CAL27). Briefly, cells $\left(2 \times 10^{4}\right)$ were seeded into the chamber coated with (for invasion) or without Matrigel (BD Biosciences, San Jose, CA) (for migration). The upper level was added with serum-free medium, while the lower level was filled with complete DMEM medium. After $24 \mathrm{~h}$ culturing, the cells migrated or invaded through the member were fixed with $4 \%$ paraformaldehyde and stained with $0.1 \%$ crystal violet. The number was counted under light microscope.

\section{Luciferase reporter assay}

For the luciferase vector of circUHRF1 and miR-526b-5p, the wild type or mutant containing the predicted miR-526b$5 p$ sequences and miR-526b-5p were cloned into the luciferase reporter vector psi-CHECK-2 (Promega, Madison, WI, USA). SCC25 cells were then cotransfected with reporter vector and miR-526b-5p or control miRNA. After $48 \mathrm{~h}$, the firefly luciferase activities were determined comparing to Renilla using Dual-Luciferase Reporter Assay System (Promega). For the luciferase promoter assay, the promoter wild type or mutant containing the predicted cMyc targeting sequences cotransfected with c-Myc plasmid or control pRL-TK Renilla plasmid into SCC25 cells using the Lipofectamine 2000 transfection reagent (Thermo Fisher Scientific). After $48 \mathrm{~h}$, the firefly activities were determined.

\section{RNA pull-down}

Biotin-labeled circUHRF1 probe and control probe (Additional file 2: Table S2) were synthesized by GenePharm 
Biotech. The extraction lysed from SCC25 cells was incubated with these probes, and then biotin-labeled RNA complex was pulled down by streptavidin magnetic beads. The beads were eluted using the elution buffer. The circUHRF1 and miR-526b-5p expression levels were examined by qRT-PCR.

\section{Chromatin immunoprecipitation (ChIP)}

ChIP was performed according to the EZ ChIP Chromatin Immunoprecipitation Kit (Millipore, Bedford, MA, USA) according to the manufacturer's protocol. Briefly, SCC25 cells were sonicated to disassemble the cross-linked chromatin DNA to be 200-1000 bp. Then, these fragments were immunoprecipitated with antibody against c-Myc (ab56, Abcam). The DNA-protein complex was precipitated and quantified using qRT-PCR using specific primers (Additional file 3: Table S3). Immunoglobulin $\mathrm{G}$ acted as a negative control.

\section{RNA-binding protein immunoprecipitation assay}

The minigenes used for the RIP assay were constructed according to previously described [27]. Briefly, the sequences, including the specific sites (GGT-rich, ESRP1 motifs) and mutant sites, of flanking intron $(11,13$ intron, $1.9 \mathrm{~kb}$ ) of circUHRF1 were synthesized and subcloned into the pLKD-CMV-EGFP vectors by GeneWiz Biotech (Guangzhou, China). The RIP assay was performed using the Magna RIP RNA-Binding Protein Immunoprecipitation Kit (Millipore, Bedford, MA) according to the manufacturer's instructions [27].

\section{In vivo xenograft experiment}

Ten male BALB/c nude mice (4 weeks old) were provided by SiPeiFu (Beijing) Biotechnology Co., Ltd. The SCC25 cells were transfected with the stable silencing plasmid (shcircUHRF1) and then subcutaneously injected with $100 \mu \mathrm{l}$ of $1 \times 10^{6}$ cell suspension at the flank. Tumor growth curve was graphed based on the tumor volumes, which was measured by vernier caliper and calculated using the formula: length $\times$ width $^{2} \times 0.5$. Tumor weight was determined after mice sacrifice. The tissue were stained with IHC staining and hematoxylin-eosin (HE) staining. The animal experiment was approved by the Ethics Committee of The Hospital of Stomatology, Tianjin Medical University.

\section{Statistical analysis}

Each experiment was repeated three times. Data were shown as means \pm standard deviation (SD). The difference within two groups was compared by $t$-test. And, the difference within multiple groups was compared one-way analysis of variance. Statistical analysis was performed using SPSS 19 (IBM, Armonk, NY, USA). $P$-value $<0.05$ was considered as statistical significance.

Acknowledgements This work was supported by National Natural Science Foundation of China (Grant/Award Numbers: 81701019, 81870763), Tianjin Science and Technology Commission General Project (Grant/Award Numbers: 18JCYBJC92400), Tianjin Stomatology Hospital Doctor/Master Key Project (Grant/Award Numbers: 2019BSZD06).

Author contributions WZ, YC, and LL perform the experiments and write the paper. XQ, JL, XH, SM, YW, ZZ, and HL act as the assists. JW, ZL, and ZW are responsible for the designing and funding collection. All authors read and approved the final paper.

\section{Compliance with ethical standards}

Conflict of interest The authors declare that they have no conflict of interest.

Publisher's note Springer Nature remains neutral with regard to jurisdictional claims in published maps and institutional affiliations.

\section{References}

1. Alves AM, Diel LF, Lamers ML. Macrophages and prognosis of oral squamous cell carcinoma: A systematic review. J Oral Pathol Med. 2018;47:460-7.

2. Safi AF, Grochau K, Drebber U, Schick V, Thiele O, Backhaus T, et al. A novel histopathological scoring system for patients with oral squamous cell carcinoma. Clinical Oral Investig. 2019. https://doi.org/10.1007/s00784-019-02804-4.

3. Zhou MY, Yang JM, Xiong XD. The emerging landscape of circular RNA in cardiovascular diseases. J Mol Cell Cardiol. 2018;122:134-9.

4. Wu J, Qi X, Liu L, Hu X, Liu J, Yang J, et al. Emerging epigenetic regulation of circular RNAs in human cancer. Mol Ther Nucleic Acids. 2019;16:589-96.

5. Wu J, Zhao W, Wang Z, Xiang X, Zhang S, Liu L. Long noncoding RNA SNHG20 promotes the tumorigenesis of oral squamous cell carcinoma via targeting miR-197/LIN28 axis. J Cell Mol Med. 2019;23:680-8.

6. Meng S, Zhou H, Feng Z, Xu Z, Tang Y, Li P, et al. CircRNA: functions and properties of a novel potential biomarker for cancer. Mol Cancer. 2017;16:94.

7. Zhao W, Ma X, Liu L, Chen Q, Liu Z, Zhang Z, et al. SNHG20: A vital lncRNA in multiple human cancers. J Cell Physiol. 2019;234:14519-25.

8. Chen L, Zhang S, Wu J, Cui J, Zhong L, Zeng L, et al. circRNA_100290 plays a role in oral cancer by functioning as a sponge of the miR-29 family. Oncogene. 2017;36:4551-61.

9. Qu S, Liu Z, Yang X, Zhou J, Yu H, Zhang R, et al. The emerging functions and roles of circular RNAs in cancer. Cancer Lett. 2018;414:301-9.

10. Zhong Y, Du Y, Yang X, Mo Y, Fan C, Xiong F, et al. Circular RNAs function as ceRNAs to regulate and control human cancer progression. Mol Cancer. 2018;17:79.

11. Huang C, Shan G. What happens at or after transcription: Insights into circRNA biogenesis and function. Transcription. 2015;6:61-64. 
12. Wilusz JE. A 360 degrees view of circular RNAs: from biogenesis to functions. Wiley Interdiscip Rev RNA. 2018;9:e1478.

13. El Marabti E, Younis I. The cancer spliceome: reprograming of alternative splicing in cancer. Front Mol Biosci. 2018;5:80.

14. Conn SJ, Pillman KA, Toubia J, Conn VM, Salmanidis M, Phillips CA, et al. The RNA binding protein quaking regulates formation of circRNAs. Cell. 2015;160:1125-34.

15. Gottgens EL, Span PN, Zegers MM. Roles and regulation of epithelial splicing regulatory proteins 1 and 2 in epithelialmesenchymal transition. Int Rev Cell Mol Biol. 2016;327:163-94.

16. Matos ML, Lapyckyj L, Rosso M, Besso MJ, Mencucci MV, Briggiler CI, et al. Identification of a novel human E-Cadherin splice variant and assessment of its effects upon EMT-related events. J Cell Physiol. 2017;232:1368-86.

17. Lekva T, Berg JP, Lyle R, Heck A, Ringstad G, Olstad OK, et al. Epithelial splicing regulator protein 1 and alternative splicing in somatotroph adenomas. Endocrinology. 2013;154:3331-43.

18. Warzecha CC, Jiang P, Amirikian K, Dittmar KA, Lu H, Shen S, et al. An ESRP-regulated splicing programme is abrogated during the epithelial-mesenchymal transition. EMBO J. 2010;29:3286-3300.

19. Ishii H, Saitoh M, Sakamoto K, Kondo T, Katoh R, Tanaka S, et al. Epithelial splicing regulatory proteins 1 (ESRP1) and 2 (ESRP2) suppress cancer cell motility via different mechanisms. J Biol Chem. 2014;289:27386-99.

20. Horiguchi K, Sakamoto K, Koinuma D, Semba K, Inoue A, Inoue $\mathrm{S}$, et al. TGF-beta drives epithelial-mesenchymal transition through deltaEF1-mediated downregulation of ESRP. Oncogene. 2012;31:3190-201.

21. Harvey SE, Xu Y, Lin X, Gao XD, Qiu Y, Ahn J, et al. Coregulation of alternative splicing by hnRNPM and ESRP1 during EMT. RNA. 2018;24:1326-38.

22. Jeong HM, Han J, Lee SH, Park HJ, Lee HJ, Choi JS, et al. ESRP1 is overexpressed in ovarian cancer and promotes switching from mesenchymal to epithelial phenotype in ovarian cancer cells. Oncogenesis. 2017;6:e389.

23. Bose R, Ain R. Regulation of transcription by circular RNAs. Adv Exp Med Biol. 2018;1087:81-94.

24. Pallavi N, Nalabolu GRK, Hiremath SKS. Bcl-2 and c-Myc expression in oral dysplasia and oral squamous cell carcinoma: An immunohistochemical study to assess tumor progression. J Oral Maxillofac Pathol. 2018;22:325-31.

25. Sambandam Y, Sundaram K, Liu A, Kirkwood KL, Ries WL, Reddy SV. CXCL13 activation of c-Myc induces RANK ligand expression in stromal/preosteoblast cells in the oral squamous cell carcinoma tumor-bone microenvironment. Oncogene. 2013;32:97-105.

26. Zeng K, He B, Yang BB, Xu T, Chen X, Xu M, et al. The prometastasis effect of circANKS1B in breast cancer. Mol Cancer. 2018;17:160.

27. Yu CY, Li TC, Wu YY, Yeh CH, Chiang W, Chuang CY, et al. The circular RNA circBIRC6 participates in the molecular circuitry controlling human pluripotency. Nat Commun. 2017; 8:1149.

28. Cui Y, Li C, Zeng C, Li J, Zhu Z, Chen W, et al. Tongmai Yangxin pills anti-oxidative stress alleviates cisplatin-induced cardiotoxicity: network pharmacology analysis and experimental evidence. Biomedicine Pharmacother. 2018;108:1081-9.

29. Ebbesen KK, Kjems J, Hansen TB. Circular RNAs: identification, biogenesis and function. Biochim Biophys Acta. 2016;1859: $163-8$.

30. Su C, Gao X, Yang W, Zhao Y, Fu X, Cui X, et al. Phosphorylation of Tudor-SN, a novel substrate of JNK, is involved in the efficient recruitment of Tudor-SN into stress granules. Biochim Biophys Acta Mol Cell Res. 2017;1864:562-71.

31. Chen LL. The biogenesis and emerging roles of circular RNAs. Nat Rev Mol Cell Biol. 2016;17:205-11.

32. Wang YF, Li BW, Sun S, Li X, Su W, Wang ZH, et al. Circular RNA expression in oral squamous cell carcinoma. Front Oncol. 2018;8:398.

33. Liang HF, Zhang XZ, Liu BG, Jia GT, Li WL. Circular RNA circ-ABCB10 promotes breast cancer proliferation and progression through sponging miR-1271. Am J Cancer Res. 2017; 7:1566-76.

34. Li S, Zhang S, Chen J. c-Myc induced upregulation of long noncoding RNA SNHG16 enhances progression and carcinogenesis in oral squamous cell carcinoma. Cancer Gene Ther. 2019. https:// doi.org/10.1038/s41417-018-0072-8.

35. Yang C, Yuan W, Yang X, Li P, Wang J, Han J, et al. Circular RNA circ-ITCH inhibits bladder cancer progression by sponging miR-17/miR-224 and regulatingp21, PTEN expression. Mol Cancer. 2018;17:19.

36. Meng J, Chen S, Han JX, Qian B, Wang XR, Zhong WL, et al. Twist1 regulates vimentin through Cul2 Circular RNA to promote EMT in hepatocellular carcinoma. Cancer Res. 2018;78: 4150-62.

37. Yang J, Liu K, Yang J, Jin B, Chen H, Zhan X, et al. PIM1 induces cellular senescence through phosphorylation of UHRF1 at Ser311. Oncogene. 2017;36:4828-42.

38. Ashwal-Fluss R, Meyer M, Pamudurti NR, Ivanov A, Bartok O, Hanan M, et al. circRNA biogenesis competes with pre-mRNA splicing. Mol Cell. 2014;56:55-66.

39. Wang R, Zhang S, Chen X, Li N, Li J, Jia R, et al. EIF4A3induced circular RNA MMP9 (circMMP9) acts as a sponge of miR-124 and promotes glioblastoma multiforme cell tumorigenesis. Mol Cancer. 2018;17:166.

40. Han J, Meng J, Wang X, Yin S, Zhang Q, Liu H, et al. YY1 complex promotes Quaking expression via super-enhancer binding during EMT of hepatocellular carcinoma. Cancer Res. 2019; 79:1451-64.

41. Li Q, Wang Y, Wu S, Zhou Z, Ding X, Shi R, et al. CircACC1 regulates assembly and activation of AMPK complex under metabolic stress. Cell Metab. 2019;30:157-173.e157. 